\title{
\%. \\ De aequationibus quarti et sexti gradus quae in theoria linearum et superficierum secundi gradus occurrunt.
}

\author{
(Auctore F. Joachimsthal, Prof. ord.)
}

\begin{abstract}
Quae olim de normalibus linearum et superficierum secundi gradus exposui theoremata, ope duarum aequationum, quarum una quarti altera autem sexti gradus, demonstrari possunt. Cum autem in utraque, quam ea de re conscripsi, commentatione geometrica, harum aequationum proprietates algebraicas consulte praetermiserim, haud inutile fore credo, formulas, quibus antea usus sum, cum Geometris nunc communicare. In fine commentationis nonnulla de aequatione generaliore $2 n t i$ gradus adjiciam, quae illas tanquam casus speciales continet.
\end{abstract}

I.

Problema de inveniendis normalibus a puncto $(\xi, \eta)$ ad conicam ad coordinatas rectangulares relatam

$$
\text { (1.) } \frac{x^{2}}{a}+\frac{y^{2}}{b}=1
$$

ductis, a resolutione aequationis (1.) atque

$$
\text { (2.) } \quad a \frac{\xi-x}{x}=b \frac{\eta-y}{y}
$$

pendet, ubi $x$ et $y$ tanquam incognitae spectandae sunt. Introducendo aliam incognitam $u$, valoribus fractionum in (2.) contentarum aequalem, habemus

atque hinc, per aequationem (1.)

$$
\left(2^{*} .\right) \quad x=\frac{a \xi}{a+u} \quad y=\frac{b \eta}{b+u},
$$

(3.) $\frac{a \xi^{2}}{(a+u)^{2}}+\frac{b \eta^{2}}{(b+u)^{2}}=1$,

sive, si brevitatis causa loco ipsarum $a \xi^{2}, b \eta^{2}$ litterae $\alpha, \beta$ scribuntur

$$
\text { (4.) } \frac{\alpha}{(a+u)^{2}}+\frac{\beta}{(b+u)^{2}}=1 \text {, }
$$

cujus aequationis biquadraticae radices signis $u_{1}, u_{2}, u_{3}, u_{4}$ denotentur. 
7. Joachimsthal, de quibusd. aequat. quarti et sexti yradus.

Ut relationes inter normales inveniantur, quae a coordinatis $\xi$, $\eta$ non pendeant, quantitates $\alpha$ et $\beta$ inter aequationes

$$
\text { (5.) }\left\{\begin{array}{l}
\frac{\alpha}{\left(a+u_{1}\right)^{2}}+\frac{\beta}{\left(b+u_{1}\right)^{2}}=1 \\
\frac{\alpha}{\left(a+u_{2}\right)^{2}}+\frac{\beta}{\left(b+u_{2}\right)^{2}}=1 \\
\frac{\alpha}{\left(a+u_{3}\right)^{2}}+\frac{\beta}{\left(b+u_{3}\right)^{2}}=1
\end{array}\right.
$$

eliminandae sunt. Aequatio, quam nota eliminationis regula obtinemus, more auctorum recentiorum, qui theoriam determinantium coluerunt, hoc modo exhiberi potest

$$
\text { (6.) }\left|\begin{array}{lll}
\frac{1}{\left(a+u_{1}\right)^{2}} & \frac{1}{\left(b+u_{1}\right)^{2}} & 1 \\
\frac{1}{\left(a+u_{2}\right)^{2}} & \frac{1}{\left(b+u_{2}\right)^{2}} & 1 \\
\frac{1}{\left(a+u_{3}\right)^{2}} & \frac{1}{\left(b+u_{3}\right)^{2}} & 1
\end{array}\right|=0 \text {. }
$$

Quum autem haec aequatio factorem supervacaneum $\left(\boldsymbol{u}_{1}-\boldsymbol{u}_{2}\right)\left(\boldsymbol{u}_{1}-\boldsymbol{u}_{3}\right)\left(\boldsymbol{u}_{2}-\boldsymbol{u}_{3}\right)$ manifesto contineat alia eruenda erit eliminationis methodus cujus ope ad relationem ab hoc incommodo liberam perveniatur.

Designando per $\varphi(a)$ functionem primi vel secundi gradus, atque ponendo

$$
\begin{aligned}
& \left(a+u_{1}\right)\left(a+u_{2}\right)\left(a+u_{3}\right)=\boldsymbol{A}, \\
& \left(b+u_{1}\right)\left(b+u_{2}\right)\left(b+u_{3}\right)=\boldsymbol{B},
\end{aligned}
$$

habemus formulam notam

$$
\frac{\varphi(a)}{A}=\frac{\varphi\left(-u_{1}\right)}{u_{2}-u_{1} \cdot u_{3}-u_{1}} \frac{1}{a+u_{1}}+\frac{\varphi\left(-u_{2}\right)}{u_{1}-u_{2} \cdot u_{3}-u_{2}} \frac{1}{a+u_{2}}+\frac{\varphi\left(-u_{3}\right)}{u_{1}-u_{3} \cdot u_{2}-u_{3}} \frac{1}{a+u_{3}}
$$

quae differentiatione respectu quantitatis $a$ in hanc

$$
\begin{gathered}
\text { (7.) } \frac{\varphi(a) A^{\prime}-\varphi^{\prime}(a) A}{A^{2}} \\
=\frac{\varphi\left(-u_{1}\right)}{u_{2}-u_{1} \cdot u_{3}-u_{1}} \frac{1}{\left(a+u_{1}\right)^{2}}+\frac{\varphi\left(-u_{2}\right)}{u_{1}-u_{2} \cdot u_{3}-u_{2}} \frac{1}{\left(a+u_{2}\right)^{2}}+\frac{\varphi\left(-u_{3}\right)}{u_{1}-u_{3} \cdot u_{2}-u_{3}} \frac{1}{\left(a+u_{3}\right)^{2}}
\end{gathered}
$$

transit. Jam multiplicando aequationes (5.) per quantitates

$$
\frac{\varphi\left(-u_{1}\right)}{u_{2}-u_{1} \cdot u_{3}-u_{1}}, \quad \frac{\varphi\left(-u_{2}\right)}{u_{1}-u_{2} \cdot u_{3}-u_{2}}, \quad \frac{\varphi\left(-u_{3}\right)}{u_{1}-u_{3} \cdot u_{2}-u_{3}}
$$

atque addendo, obtinemus ope aequationis (\%.) 
7. Joachimsthal, de quibusd. aequat. quarti et sexti gradus.

$$
\text { (8.) } \begin{aligned}
& \frac{\alpha}{\boldsymbol{A}^{2}}\left\{\varphi(\boldsymbol{a}) \boldsymbol{A}^{\prime}-\varphi^{\prime}(\boldsymbol{a}) \boldsymbol{A}\right\}+\frac{\beta}{\boldsymbol{B}^{2}}\left\{\varphi(\boldsymbol{b}) \boldsymbol{B}^{\prime}-\varphi^{\prime}(\boldsymbol{b}) \boldsymbol{B}\right\} \\
= & \frac{\varphi\left(-u_{1}\right)}{u_{2}-u_{1} \cdot u_{3}-u_{1}}+\frac{\varphi\left(-u_{2}\right)}{u_{1}-u_{2} \cdot u_{3}-u_{2}}+\frac{\varphi\left(-u_{3}\right)}{u_{1}-u_{3} \cdot u_{2}-u_{3}} .
\end{aligned}
$$

Sit $\varphi(a)$ functio linearis $=a+\lambda$, ubi $\lambda$ quantitatem constantem denotat. Pars dextra aequationis praecedentis secundum formulam Eulerianam evanescere debet, atque habemus

$$
\text { (9.) } \quad \frac{\alpha}{A^{2}}\left\{\varphi(\boldsymbol{a}) \boldsymbol{A}^{\prime}-\varphi^{\prime}(\boldsymbol{a}) \boldsymbol{A}\right\}+\frac{\beta}{\boldsymbol{B}^{2}}\left\{\varphi(\boldsymbol{b}) \boldsymbol{B}^{\prime}-\varphi^{\prime}(\boldsymbol{b}) \boldsymbol{B}\right\}=\mathbf{0} \text {. }
$$

Sed quantitas arbitraria $\lambda$ ita determinari potest, ut sit

$$
\text { (10.) } \varphi(\boldsymbol{a}) \boldsymbol{A}^{\prime}-\varphi^{\prime}(\boldsymbol{a}) \boldsymbol{A}=0, \quad \text { sive } \frac{1}{a+\lambda}=\frac{\boldsymbol{A}^{\prime}}{\boldsymbol{A}}
$$

qua relatione (9.) in hancce transit

$$
\text { (11.) } \varphi(b) \boldsymbol{B}^{\prime}-\varphi^{\prime}(\boldsymbol{b}) \boldsymbol{B}=0, \quad \text { sive } \frac{1}{b+\lambda}=\frac{\boldsymbol{B}^{\prime}}{\boldsymbol{B}} \text {. }
$$

Substitutis iterum valoribus functionum $\boldsymbol{A}$ et $\boldsymbol{B}$, loco duarum aequationum praecedentium habentur sequentes

$$
\left\{\begin{array}{l}
\frac{1}{a+\lambda}=\frac{1}{a+u_{1}}+\frac{1}{a+u_{2}}+\frac{1}{a+u_{3}} \\
\frac{1}{b+\lambda}=\frac{1}{b+u_{1}}+\frac{1}{b+u_{2}}+\frac{1}{b+u_{3}} .
\end{array}\right.
$$

His calculis eliminatio duarum quantitatum $\alpha$ et $\beta$ e tribus aequationibus. (5.) ad eliminationem unius quantitatis $\lambda$ e duabus aequationibus (12.) reducta est. Jam subtrahendo aequationes (12.) obtinemus, suppresso factore $a-b$,

$$
\frac{1}{(a+\lambda)(b+\lambda)}=\frac{1}{\left(a+u_{1}\right)\left(b+u_{1}\right)}+\frac{1}{\left(a+u_{2}\right)\left(b+u_{2}\right)}+\frac{1}{\left(a+u_{3}\right)\left(b+u_{3}\right)}
$$

et e combinatione satis obvia aequationum (12.) et (13.) haec aequatio finalis respectu radicum $u_{1}, u_{2}, u_{3}$ omnino symmetrica

$$
\begin{aligned}
& \frac{1}{\left(a+u_{2}\right)\left(b+u_{3}\right)}+\frac{1}{\left(a+u_{3}\right)\left(b+u_{2}\right)}+\frac{1}{\left(a+u_{3}\right)\left(b+u_{1}\right)} \\
+ & \frac{1}{\left(a+u_{1}\right)\left(b+u_{3}\right)}+\frac{1}{\left(a+u_{1}\right)\left(b+u_{2}\right)}+\frac{1}{\left(a+u_{2}\right)\left(b+u_{1}\right)}=0
\end{aligned}
$$

derivatur. Calculo praecedenti igitur nacti sumus

Theorema I. Designando quantitatem $\frac{1}{\left(a+u_{1}\right)\left(b+u_{2}\right)}+\frac{1}{\left(a+u_{2}\right)\left(b+u_{1}\right)}$ characteristica $(1,2)$, inter ternas radices $u_{1}, u_{2}, u_{3}$ aequationis biquaJournal f. d. M. Bd. LIIl. Heft 2. 
draticae

relatio intercedit

$$
\text { (4.) } \frac{\alpha}{(a+u)^{2}}+\frac{\beta}{(b+u)^{2}}=1
$$

$$
\text { (14*) } \quad(2,3)+(1,3)+(1,2)=0 .
$$

Qua ex aequatione tres aliae, illi $\left(14^{*}\right.$.) analogae, permutatione radicum deduci possunt, scilicet

$$
\begin{aligned}
(15 .) & (2,4)+(1,4)+(1,2)=0, \\
\left(15^{*} .\right) & (1,4)+(1,3)+(3,4)=0, \\
\left(15^{* *} .\right) & (2,4)+(2,3)+(3,4)=0 .
\end{aligned}
$$

Formando combinationem $\left(14^{*}.\right)+(15)=.\left(15^{*}.\right)+\left(15^{* *}\right.$.) prodit alia aequatio haud inelegans

$$
\text { (16.) }(1,2)=(3,4)
$$

unde fluit

Theorema II. Inter quatuor radices aequationis $\frac{\alpha}{(a+u)^{2}}+\frac{\beta}{(b+u)^{2}}=1$ valet relatio

$$
\frac{1}{\left(a+u_{1}\right)\left(b+u_{2}\right)}+\frac{1}{\left(a+u_{2}\right)\left(b+u_{1}\right)}=\frac{1}{\left(a+u_{3}\right)\left(b+u_{4}\right)}+\frac{1}{\left(a+u_{4}\right)\left(b+u_{3}\right)} .
$$

Cujus theorematis adjumento sex expressiones

$$
(1,2),(1,3),(1,4),(2,3),(2,4),(3,4),
$$

quum sit $(1,2)=(3,4),(1,3)=(2,4),(1,4)=(2,3)$ ad tres diversas $(1,2),(1,3),(1,4)$ reducuntur quae tanquam radices aequationis cubicae, formae

$$
\boldsymbol{U}^{3}+\lambda^{\prime} \boldsymbol{U}+\lambda^{\prime \prime}=\mathbf{0}
$$

inveniri possunt, ubi $\lambda^{\prime}, \lambda^{\prime \prime}$ ipsarum $\alpha, \beta, a, b$ sunt functiones algebraicae facili negotio eruendae.

Observatio. Aequatio fundamentalis (4.) in formam trigonometricam redigi potest, ponendo

unde prodit

$$
\frac{\sqrt{ } \alpha}{a+u}=\cos \varphi, \quad \frac{\sqrt{ } \beta}{b+u}=\sin \varphi
$$

$$
\frac{\sqrt{ } \alpha}{\cos \varphi}-\frac{\sqrt{ } \beta}{\sin \varphi}=a-b,
$$

sive

$$
l \sin \varphi+m \cos \varphi=\sin \varphi \cos \varphi, \quad \text { ubi } \quad l=\frac{\sqrt{ } a}{a-b}, \quad m=-\frac{\sqrt{ } \beta}{a-b} .
$$


7. Joachimsthal, de quibusd. aequat. quarti et sexti gradus.

Quae igitur de aequatione biquadratica (4.) supra invenimus theoremata, nunc ita audiunt.

„Inter radices $\varphi_{1}, \varphi_{2}, \varphi_{3}, \varphi_{4}$ aequationis $l \sin \varphi+m \cos \varphi=\sin \varphi \cos \varphi$ ,intercedunt relationes

$$
\begin{gathered}
\sin \left(\varphi_{1}+\varphi_{2}\right)+\sin \left(\varphi_{1}+\varphi_{3}\right)+\sin \left(\varphi_{2}+\varphi_{3}\right)=0 \\
\varphi_{1}+\varphi_{2}+\varphi_{3}+\varphi_{4}=(2 n+1) \pi ; \quad n=\text { num. int." }
\end{gathered}
$$

Interpretationem geometricam relationis (14.) infra examinabimus.

II.

Perinde problema ducendi lineas normales a puncto $(\xi, \eta, \zeta)$ ad superficiem

$$
\frac{x^{2}}{a}+\frac{y^{2}}{b}+\frac{z^{2}}{c}=1
$$

a resolutione aequationis sexti gradus

sive

$$
\frac{a \xi^{2}}{(a+u)^{2}}+\frac{b \eta^{2}}{(b+u)^{2}}+\frac{c \zeta^{2}}{(c+u)^{2}}=1
$$

$$
\frac{a}{(a+u)^{2}}+\frac{\beta}{(b+u)^{2}}+\frac{\gamma}{(c+u)^{2}}=1
$$

pendet, ubi brevitatis gratia $\alpha, \beta, \gamma$ loco ipsarum $a \xi^{2}, b \eta^{2}, c \zeta^{2}$ scripsimus. Ut proprietates normalium per idem punctum $(\xi, \eta, \zeta)$ transeuntium detegantur, inter quatuor aequationes

$$
\left\{\begin{array}{l}
\frac{\alpha}{\left(a+u_{1}\right)^{2}}+\frac{\beta}{\left(b+u_{1}\right)^{2}}+\frac{\gamma}{\left(c+u_{1}\right)^{2}}=1 \\
\frac{\alpha}{\left(a+u_{2}\right)^{2}}+\frac{\beta}{\left(b+u_{2}\right)^{2}}+\frac{\gamma}{\left(c+u_{2}\right)^{2}}=1 \\
\frac{\alpha}{\left(a+u_{3}\right)^{2}}+\frac{\beta}{\left(b+u_{3}\right)^{2}}+\frac{\gamma}{\left(c+u_{3}\right)^{2}}=1 \\
\frac{\alpha}{\left(a+u_{4}\right)^{2}}+\frac{\beta}{\left(b+u_{4}\right)^{2}}+\frac{\gamma}{\left(c+u_{4}\right)^{2}}=1
\end{array}\right.
$$

quantitates $\alpha, \beta, \gamma$ eliminandae sunt, ita quidem ut aequatio finalis a producto differentiarum $u_{1}-u_{2} \cdot u_{1}-u_{3} \cdot u_{1}-u_{4} \cdot u_{2}-u_{3} \cdot u_{2}-u_{4} . u_{3}-u_{4}$ sit libera. Scribendo

$$
\text { (20.) } \quad\left\{\begin{array}{c}
\left(a+u_{1}\right)\left(a+u_{2}\right)\left(a+u_{3}\right)\left(a+u_{4}\right)=A \\
\left(b+u_{1}\right)\left(b+u_{2}\right)\left(b+u_{3}\right)\left(b+u_{4}\right)=B \\
\left(c+u_{1}\right)\left(c+u_{2}\right)\left(c+u_{3}\right)\left(c+u_{4}\right)=C \\
20^{*}
\end{array}\right.
$$


atque statuendo $\varphi(a)=(a+\lambda)(a+\mu)$, habetur

$$
\frac{\varphi(a)}{A}=\frac{\varphi\left(-u_{1}\right)}{u_{2}-u_{1} \cdot u_{3}-u_{1} \cdot u_{4}-u_{1}} \frac{1}{a+u_{1}}+\cdots
$$

unde, differentiatione respectu ipsius $a$ instituta, prodit aequatio

$$
\frac{\varphi(a) A^{\prime}-\varphi^{\prime}(a) A}{A^{2}}=\Sigma \frac{\varphi\left(-u_{1}\right)}{u_{2}-u_{1} \cdot u_{3}-u_{1} \cdot u_{4}-u_{1}} \frac{1}{\left(a+u_{1}\right)^{2}} .
$$

Cujus formulae ope, si aequationes (19.) ex ordine factoribus

$$
\begin{aligned}
& \frac{\varphi\left(-u_{1}\right)}{u_{2}-u_{1} \cdot u_{3}-u_{1} \cdot u_{4}-u_{1}}, \quad \frac{\varphi\left(-u_{2}\right)}{u_{1}-u_{2} \cdot u_{3}-u_{2} \cdot u_{4}-u_{2}}, \frac{\varphi\left(-u_{3}\right)}{u_{1}-u_{3} \cdot u_{2}-u_{3} \cdot u_{4}-u_{3}}, \\
& \frac{\varphi\left(-u_{4}\right)}{u_{1}-u_{4} \cdot u_{2}-u_{4} \cdot u_{3}-u_{4}}
\end{aligned}
$$

multiplicantur, obtinemus addendo

$$
\begin{aligned}
\frac{\boldsymbol{\alpha}}{\boldsymbol{A}^{2}}\left\{\varphi(\boldsymbol{a}) \boldsymbol{A}^{\prime}-\varphi^{\prime}(\boldsymbol{a}) \boldsymbol{A}\right\} & +\frac{\beta}{\boldsymbol{B}^{2}}\left\{\varphi(\boldsymbol{b}) \boldsymbol{B}^{\prime}-\varphi^{\prime}(\boldsymbol{b}) \boldsymbol{B}\right\}+\frac{\gamma}{\boldsymbol{C}^{2}}\left\{\varphi(\boldsymbol{c}) \boldsymbol{C}^{\prime}-\varphi^{\prime}(\boldsymbol{c}) \boldsymbol{C}\right\} \\
= & \sum \frac{\varphi\left(-u_{1}\right)}{u_{2}-u_{1} \cdot u_{3}-u_{1} \cdot u_{4}-u_{1}} .
\end{aligned}
$$

Quum functio $\varphi$ secundum gradum non transgrediatur, pars dextra hujus formulae evanescit; habemus itaque

$$
\frac{\alpha}{\boldsymbol{A}^{2}}\left\{\varphi(\boldsymbol{a}) \boldsymbol{A}^{\prime}-\varphi^{\prime}(\boldsymbol{a}) \boldsymbol{A}\right\}+\frac{\beta}{\boldsymbol{B}^{2}}\left\{\varphi(\boldsymbol{b}) \boldsymbol{B}^{\prime}-\varphi^{\prime}(\boldsymbol{b}) \boldsymbol{B}\right\}+\frac{\gamma}{\boldsymbol{C}^{2}}\left\{\varphi(\boldsymbol{c}) \boldsymbol{C}^{\prime}-\varphi^{\prime}(\boldsymbol{c}) \boldsymbol{C}\right\}=\mathbf{0}
$$

Quantilates $\lambda$ et $\mu$, quae in functione $\varphi$ inveniuntur, quippe quae omnino arbitrariae sint, ita determinari possunt, ut ipsarum $\frac{\alpha}{A^{2}}, \frac{\beta}{B^{2}}$ coefficientes evanescant. Quo facto, aequatio (22.) in tres alias discerpitur,

$$
\varphi(\boldsymbol{a}) \boldsymbol{A}^{\prime}-\varphi^{\prime}(\boldsymbol{a}) \boldsymbol{A}=0, \quad \varphi(\boldsymbol{b}) \boldsymbol{B}^{\prime}-\varphi^{\prime}(\boldsymbol{b}) \boldsymbol{B}=0, \quad \varphi(\boldsymbol{c}) \boldsymbol{C}^{\prime}-\varphi^{\prime}(\boldsymbol{c}) \boldsymbol{C}=\mathbf{0}
$$

sive in

(23.)

$$
\left\{\begin{array}{l}
\frac{1}{a+\lambda}+\frac{1}{a+\mu}=\frac{1}{a+u_{1}}+\frac{1}{a+u_{2}}+\frac{1}{a+u_{3}}+\frac{1}{a+u_{4}} \\
\frac{1}{b+\lambda}+\frac{1}{b+\mu}=\frac{1}{b+u_{1}}+\frac{1}{b+u_{2}}+\frac{1}{b+u_{3}}+\frac{1}{b+u_{4}} \\
\frac{1}{c+\lambda}+\frac{1}{c+\mu}=\frac{1}{c+u_{1}}+\frac{1}{c+u_{2}}+\frac{1}{c+u_{3}}+\frac{1}{c+u_{4}} .
\end{array}\right.
$$

Reduximus itaque eliminationem ipsarum $\alpha, \beta, \gamma$ e quatuor aequationibus (19.) ad eliminationem duarum quantitatum $\lambda$ et $\mu$ e tribus aequationibus (23.). Quae eliminatio, quamvis illa, quam supra (I.) tractavimus, multo complicatior, haud ineleganter hoc modo perficitur. 
Subtrahendo binas aequationes (23.) habemus

(24.)

$$
\left\{\begin{array}{c}
\frac{1}{(a+\lambda)(b+\lambda)}+\frac{1}{(a+\mu)(b+\mu)} \\
=\frac{1}{\left(a+u_{1}\right)\left(b+u_{1}\right)}+\frac{1}{\left(a+u_{2}\right)\left(b+u_{2}\right)}+\frac{1}{\left(a+u_{3}\right)\left(b+u_{3}\right)}+\frac{1}{\left(a+u_{4}\right)\left(b+u_{4}\right)} \\
\frac{1}{(b+\lambda)(c+\lambda)}+\frac{1}{(b+\mu)(c+\mu)} \\
=\frac{1}{\left(b+u_{1}\right)\left(c+u_{1}\right)}+\frac{1}{\left(b+u_{2}\right)\left(c+u_{2}\right)}+\frac{1}{\left(b+u_{3}\right)\left(c+u_{3}\right)}+\frac{1}{\left(b+u_{4}\right)\left(c+u_{4}\right)} \\
=\frac{1}{(a+\lambda)(c+\lambda)}+\frac{1}{(a+\mu)(c+\mu)} \\
=\frac{1}{\left(a+u_{1}\right)\left(c+u_{1}\right)}+\frac{1}{\left(a+u_{2}\right)\left(c+u_{2}\right)}+\frac{1}{\left(a+u_{3}\right)\left(c+u_{3}\right)}+\frac{1}{\left(a+u_{4}\right)\left(c+u_{4}\right)}
\end{array}\right.
$$

et multiplicando aequationes (23.) ex ordine per

$$
\frac{1}{b-a \cdot c-a}, \quad \frac{1}{a-b \cdot c-b}, \quad \frac{1}{a-c . b-c}
$$

atque addendo, habetur

$$
\begin{gathered}
\text { (25.) } \frac{1}{(a+\lambda)(b+\lambda)(c+\lambda)}+\frac{1}{(a+\mu)(b+\mu)(c+\mu)} \\
=\frac{1}{\left(a+u_{1}\right)\left(b+u_{1}\right)\left(c+u_{1}\right)}+\cdots+\frac{1}{\left(a+u_{4}\right)\left(b+u_{4}\right)\left(c+u_{4}\right)} .
\end{gathered}
$$

Jam procul dubio lectorem accuratius examinantem non fugit, calculos, quorum adjumento ex aeq. (12.) et (13.) quantitatem $\lambda$ eliminavimus, hac formula concinne exhiberi posse

$$
\begin{gathered}
\left(\xi_{1}+\xi_{2}+\cdots+\xi_{n}\right)\left(\eta_{1}+\eta_{2}+\cdots+\eta_{n}\right)-\left(\xi_{1} \eta_{1}+\xi_{2} \eta_{2}+\cdots+\xi_{n} \eta_{n}\right) \\
=\Sigma\left(\xi_{1} \eta_{2}+\xi_{2} \eta_{1}\right) .
\end{gathered}
$$

Si $n=1$, pars laeva in nihilum abit; si autem $n \equiv 2$, summa ad dextram $\frac{n(n-1)}{2}$ summas binarias ut $\xi_{1} \eta_{2}+\xi_{2} \eta_{1}$ continet.

Est autem formula (26.) prima in serie quadam infinita formularum, quarum secunda hoc modo scribi potest:

$$
\text { (27.) } \begin{aligned}
& \Sigma \xi_{1} \Sigma \eta_{1} \Sigma \zeta_{1}-\Sigma \xi_{1} \Sigma \eta_{1} \zeta_{1}-\Sigma \eta_{1} \Sigma \xi_{1} \zeta_{1}-\Sigma \zeta_{1} \Sigma \xi_{1} \eta_{1}+2 \Sigma \xi_{1} \eta_{1} \zeta_{1} \\
& =\Sigma\left(\xi_{1} \eta_{2} \zeta_{3}+\xi_{1} \eta_{3} \zeta_{2}+\xi_{2} \eta_{3} \zeta_{1}+\xi_{2} \eta_{1} \zeta_{3}+\xi_{3} \eta_{1} \zeta_{2}+\xi_{3} \eta_{2} \zeta_{1}\right) .
\end{aligned}
$$

Summae $\Sigma$ in membro primo aequationis (27.) $n$ terminos, summa autem in membro secundo $\frac{n(n-1)(n-2)}{6}$ summas partiales senarias continet; si autem $n=1$, aut $n=2$, primum membrum evanescit. 
Habemus ex. gr. pro $n=3$

(28.)

$$
\begin{gathered}
\left(\xi_{1}+\xi_{2}+\xi_{3}\right)\left(\eta_{1}+\eta_{2}+\eta_{3}\right)\left(\zeta_{1}+\zeta_{2}+\zeta_{3}\right)-\left(\xi_{1}+\xi_{2}+\xi_{3}\right)\left(\eta_{1} \zeta_{1}+\eta_{2} \zeta_{2}+\eta_{3} \zeta_{3}\right) \\
-\left(\eta_{1}+\eta_{2}+\eta_{3}\right)\left(\xi_{1} \zeta_{1}+\xi_{2} \zeta_{2}+\xi_{3} \zeta_{3}\right)-\left(\zeta_{1}+\zeta_{2}+\zeta_{3}\right)\left(\xi_{1} \eta_{1}+\xi_{2} \eta_{2}+\xi_{3} \eta_{3}\right) \\
+2\left(\xi_{1} \eta_{1} \zeta_{1}+\xi_{2} \eta_{2} \zeta_{2}+\xi_{3} \eta_{3} \zeta_{3}\right) \\
=\xi_{1} \eta_{2} \zeta_{3}+\xi_{1} \eta_{3} \zeta_{2}+\xi_{2} \eta_{1} \zeta_{3}+\xi_{2} \eta_{3} \zeta_{1}+\xi_{3} \eta_{1} \zeta_{2}+\xi_{3} \eta_{2} \zeta_{1}
\end{gathered}
$$

unde fluit aequatio identica

$$
\begin{gathered}
\left(\xi_{1}+\xi_{2}\right)\left(\eta_{1}+\eta_{2}\right)\left(\zeta_{1}+\zeta_{2}\right)-\left(\xi_{1}+\xi_{2}\right)\left(\eta_{1} \zeta_{1}+\eta_{2} \zeta_{2}\right)-\left(\eta_{1}+\eta_{2}\right)\left(\xi_{1} \zeta_{1}+\xi_{2} \zeta_{2}\right) \\
-\left(\zeta_{1}+\zeta_{2}\right)\left(\xi_{1} \eta_{1}+\xi_{2} \eta_{2}\right)+2\left(\xi_{1} \eta_{1} \zeta_{1}+\xi_{2} \eta_{2} \zeta_{2}\right)=0
\end{gathered}
$$

Quibus expositis ad eliminationem ipsarum $\lambda$ et $\mu$ progrediamur. Formetur hunc ad finem expressio

$$
\begin{aligned}
& \left(\frac{1}{a+\lambda}+\frac{1}{a+\mu}\right)\left(\frac{1}{b+\lambda}+\frac{1}{b+\mu}\right)\left(\frac{1}{c+\lambda}+\frac{1}{c+\mu}\right) \\
- & \left(\frac{1}{a+\lambda}+\frac{1}{a+\mu}\right)\left(\frac{1}{(b+\lambda)(c+\lambda)}+\frac{1}{(b+\mu)(c+\mu)}\right) \\
- & \left(\frac{1}{b+\lambda}+\frac{1}{b+\mu}\right)\left(\frac{1}{(a+\lambda)(c+\lambda)}+\frac{1}{(a+\mu)(c+\mu)}\right) \\
- & \left(\frac{1}{c+\lambda}+\frac{1}{c+\mu}\right)\left(\frac{1}{(a+\lambda)(b+\lambda)}+\frac{1}{(a+\mu)(b+\mu)}\right) \\
+ & 2\left(\frac{1}{(a+\lambda)(b+\lambda)(c+\lambda)}+\frac{1}{(a+\mu)(b+\mu)(c+\mu)}\right),
\end{aligned}
$$

quae secundum formulam (29.) identice evanescit. Substitutis autem valoribus summarum $\frac{1}{a+\lambda}+\frac{1}{a+\mu}, \frac{1}{b+\lambda}+\frac{1}{b+\mu}$, etc., supra inventis (cf. formulas (23.), (24), (25.)), obtinemus ope formulae (27.) quaesitam aequationem finalem respectu radicum $u_{1}, u_{2}, u_{3}, u_{4}$ omnino symmetricam, quae brevitatis causa

$$
\text { (30.) } \quad 0=\Sigma \frac{1}{\left(a+u_{1}\right)\left(b+u_{2}\right)\left(c+u_{3}\right)}
$$

scribi potest. Signum $\Sigma$ viginti quatuor terminos continet, e termino primo

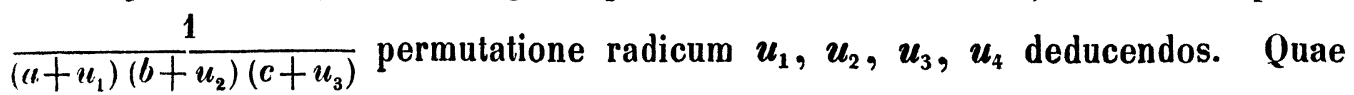
nunc inventa sunt, hoc modo enuntiari possunt.

Theorema III. Designando quantitatem

$$
\begin{gathered}
\frac{1}{\left(a+u_{1}\right)\left(b+u_{2}\right)\left(c+u_{3}\right)}+\frac{1}{\left(a+u_{1}\right)\left(b+u_{3}\right)\left(c+u_{2}\right)}+\frac{1}{\left(a+u_{2}\right)\left(b+u_{1}\right)\left(c+u_{3}\right)} \\
+\frac{1}{\left(a+u_{2}\right)\left(b+u_{3}\right)\left(c+u_{1}\right)}+\frac{1}{\left(a+u_{3}\right)\left(b+u_{1}\right)\left(c+u_{2}\right)}+\frac{1}{\left(a+u_{3}\right)\left(b+u_{2}\right)\left(c+u_{1}\right)}
\end{gathered}
$$


7. Joachimsthal, de quibusd. aequat. quarti et sexti gradus.

characteristica $(1,2,3)$, inter quaternas radices $u_{1}, u_{2}, u_{3}, u_{4}$ aequationis

intercedit relatio

$$
\text { (18.) } \frac{\alpha}{(a+u)^{2}}+\frac{\beta}{(b+u)^{2}}+\frac{\gamma}{(c+u)^{2}}=1
$$

$$
\text { (31.) }(2,3,4)+(1,3,4)+(1,2,4)+(1,2,3)=0 \text {. }
$$

Quae propositio illi analoga est quam supra demonstravimus. Similiter, ut sex quantitates in (I.) signis $(1,2),(1,3),(1,4),(2,3),(2,4),(3,4)$ denotatas ad tres diversas reduci posse docuimus, nunc reductionem viginti qnantitatum $(1,2,3),(1,2,4) \ldots(4,5,6)$ ad decem explicabimus.

Derivantur mutatione radicum e formula (31.) relationes

$$
\begin{array}{ll}
\left(31^{\text {a }} .\right) & (2,3,5)+(1,3,5)+(1,2,5)+(1,2,3)=0 \\
\left(31^{\text {b }}\right) & (2,4,5)+(1,4,5)+(1,2,5)+(1,2,4)=0 \\
\left(31^{\text {c }} .\right) & (3,4,5)+(1,4,5)+(1,3,5)+(1,3,4)=0 \\
\left(31^{\text {d }}\right) & (2,3,4)+(2,3,5)+(2,4,5)+(3,4,5)=0 \\
\left(31^{\text {e. }}\right) & (1,2,3)+(1,2,6)+(1,3,6)+(2,3,6)=0 .
\end{array}
$$

Formando combinationem $(31)+.\left(31^{\mathrm{a}}.\right)=\left(31^{\mathrm{b}}.\right)+\left(31^{\mathrm{c}}.\right)+\left(31^{\mathrm{d}}.\right)$, habemus

$$
\text { (32.) } \begin{aligned}
(1,2,3) & =(1,4,5)+(2,4,5)+(3,4,5), \text { unde } \\
(1,2,3) & =(1,4,6)+(2,4,6)+(3,4,6) \\
(1,2,3) & =(1,5,6)+(2,5,6)+(3,5,6), \text { atque similiter } \\
(4,5,6) & =(1,2,4)+(1,2,5)+(1,2,6) \\
(4,5,6) & =(1,3,4)+(1,3,5)+(1,3,6) \\
(4,5,6) & =(2,3,4)+(2,3,5)+(2,3,6) .
\end{aligned}
$$

Quarum aequationum ratione habita, summa $(31)+.\left(31^{\mathrm{a}}.\right)+\left(31^{\mathrm{e}}.\right)$ mutatur in (33.) $(1,2,3)+(4,5,6)=0$.

Nacti igitur sumus hanc propositionem

Theorema IV. Denotando per $u_{1}, u_{2}, u_{3}$ tres radices aequationis sexti gradus

$$
\text { (10.) } \frac{\alpha}{(a+u)^{2}}+\frac{\beta}{(b+u)^{2}}+\frac{\gamma}{(c+u)^{2}}=1 \text {, }
$$

aequatio vigesimi gradus, cui omnes valores diversi expressionis

$$
\begin{aligned}
\boldsymbol{U} & =\frac{1}{\left(a+u_{1}\right)\left(b+u_{2}\right)\left(c+u_{3}\right)}+\frac{1}{\left(a+u_{1}\right)\left(b+u_{3}\right)\left(c+u_{2}\right)}+\frac{1}{\left(a+u_{2}\right)\left(b+u_{1}\right)\left(c+u_{3}\right)} \\
& +\frac{1}{\left(a+u_{2}\right)\left(b+u_{3}\right)\left(c+u_{1}\right)}+\frac{1}{\left(a+u_{3}\right)\left(b+u_{1}\right)\left(c+u_{2}\right)}+\frac{1}{\left(a+u_{3}\right)\left(b+u_{2}\right)\left(c+u_{1}\right)}
\end{aligned}
$$

satisfaciunt, potestatibus imparibus ipsius $U$ caret. 
Antequam aequationis generalis $\frac{\alpha_{1}}{\left(a_{1}+u\right)^{2}}+\frac{\alpha_{2}}{\left(a_{2}+u\right)^{2}}+\cdots+\frac{\alpha_{n}}{\left(a_{n}+u\right)^{2}}=1$ proprietates deducemus, nonnulla, ne formularum nexus nimis interrumpatur, de transformatione summae $\Sigma \alpha_{1} \beta_{2} \gamma_{3} \ldots \mu_{n}$ addantur.

\section{III.}

Lemma. „Datis $n$ seriebus quantitatum

$$
\begin{aligned}
& \begin{array}{lllll}
\alpha_{1} & \alpha_{2} & \ldots & \alpha_{i}
\end{array} \\
& \beta_{1} \beta_{2} \ldots \beta_{i} \\
& \begin{array}{llll}
\lambda_{1} & \lambda_{2} & \ldots & \lambda_{i}
\end{array} \\
& \begin{array}{llll}
\mu_{1} & \mu_{2} & \ldots & \mu_{i}
\end{array} \\
& \text { summa } \Sigma \alpha_{1} \beta_{2} \gamma_{3} \ldots \mu_{n}
\end{aligned}
$$

„quae $i(i-1)(i-2) \ldots(i-n+1)$ terminos continet, ope summarum

$$
\text { (34.) }\left\{\begin{array}{cccccc}
\Sigma \alpha_{1} & \Sigma \beta_{1} & \Sigma \gamma_{1} & \ldots & \Sigma \mu_{1} \\
\Sigma \alpha_{1} \beta_{1} & \Sigma \alpha_{1} \gamma_{1} & \ldots & \Sigma \lambda_{1} \mu_{1} \\
\Sigma \alpha_{1} \beta_{1} \gamma_{1} & \Sigma \alpha_{1} \beta_{1} \delta_{1} & \ldots & \Sigma \varkappa_{1} \lambda_{1} \mu_{1} \\
\vdots & & & \\
& \Sigma \alpha_{1} \beta_{1} \gamma_{1} & \ldots & \mu_{1}
\end{array}\right.
$$

"quarum singulae $i$ terminis compositae sunt, exprimi potest, ita ut sit

$$
\text { (35.) } \Sigma \alpha_{1} \beta_{2} \gamma_{3} \ldots \mu_{n}=\boldsymbol{J} \text {, }
$$

„ubi $\boldsymbol{J}$ summarum (34.) functionem integram denotat. Dicimus insuper, „expressionem $\boldsymbol{J}$ pro $\boldsymbol{i} \overline{\bar{\chi}} \boldsymbol{n}-1$ in nihilum abire.

Quod lemma, facile ad inveniendum difficile autem ad explicandum, cum formula de reductione expressionis $\Sigma x_{1}^{\alpha_{1}} x_{2}^{\alpha_{2}} \ldots x_{n}^{\alpha_{n}}$ ad summas potestatum radicum $x_{1}, x_{2}, \ldots x_{i}$, si ad modum demonstrandi solum spectas, prorsus congruit. Evolutio casuum $i=2,=3,=4$, quorum priores jam supra indicavimus, hic sufficiet (cf. form. 26 et 27). Habetur

$$
\text { (26.) } \Sigma \alpha_{1} \beta_{2}=\Sigma \alpha_{1} \Sigma \beta_{1}-\Sigma \alpha_{1} \beta_{1}
$$

unde mutliplicando per $\Sigma \gamma_{1}$

Est autem

$$
\text { (36.) } \Sigma \alpha_{1} \beta_{2} \Sigma \gamma_{1}=\Sigma \alpha_{1} \Sigma \beta_{1} \Sigma \gamma_{1}-\Sigma \alpha_{1} \beta_{1} \Sigma \gamma_{1} .
$$

$$
\left(36^{*} .\right) \quad \Sigma \alpha_{1} \beta_{2} \Sigma \gamma_{1}=\Sigma \alpha_{1} \beta_{2} \gamma_{3}+\Sigma \alpha_{1} \gamma_{1} \beta_{2}+\Sigma \alpha_{2} \beta_{1} \gamma_{1},
$$


atque iisdem, quibus formula (26.) demonstratur, ratiociniis

$$
\begin{aligned}
& \Sigma \alpha_{1} \gamma_{1} \beta_{2}=\Sigma \alpha_{1} \gamma_{1} \Sigma \beta_{1}-\Sigma \alpha_{1} \beta_{1} \gamma_{1} \\
& \Sigma \alpha_{2} \beta_{1} \gamma_{1}=\Sigma \beta_{1} \gamma_{1} \Sigma \alpha_{1}-\Sigma \alpha_{1} \beta_{1} \gamma_{1} .
\end{aligned}
$$

His valoribus in formulis (36.) et $\left(36^{*}\right.$.) substitutis, derivatur

(37.) $\Sigma \alpha_{1} \beta_{2} \gamma_{3}=\Sigma \alpha_{1} \Sigma \beta_{1} \Sigma \gamma_{1}-\Sigma \alpha_{1} \Sigma \beta_{1} \gamma_{1}-\Sigma \beta_{1} \Sigma \gamma_{1} \alpha_{1}-\Sigma \gamma_{1} \Sigma \alpha_{1} \beta_{1}$

$$
2 \Sigma \alpha_{1} \beta_{1} \gamma_{1} \text {. }
$$

Perinde reductio ipsius $\Sigma \alpha_{1} \beta_{2} \gamma_{3} \delta_{4}$ peragitur. Nam multiplicando aequationem praecedentem per $\Sigma \delta_{1}$, obtinemus

(37*) $\Sigma \delta_{1} \Sigma \alpha_{1} \beta_{2} \gamma_{3}=\Sigma \alpha_{1} \Sigma \beta_{1} \Sigma \gamma_{1} \Sigma \delta_{1}-\Sigma \alpha_{1} \Sigma \delta_{1} \Sigma \beta_{1} \gamma_{1}-\Sigma \beta_{1} \Sigma \delta_{1} \Sigma \gamma_{1} \alpha_{1}$

$$
\Sigma \gamma_{1} \Sigma \delta_{1} \Sigma \alpha_{1} \beta_{1}+2 \Sigma \delta_{1} \Sigma \alpha_{1} \beta_{1} \gamma_{1} \text {. }
$$

Est autem

(37**) $\Sigma \delta_{1} \Sigma \alpha_{1} \beta_{2} \gamma_{3}=\Sigma \alpha_{1} \beta_{2} \gamma_{3} \delta_{4}+\Sigma \alpha_{1} \delta_{1} \beta_{2} \gamma_{3}+\Sigma \beta_{1} \delta_{1} \alpha_{2} \gamma_{3}+\Sigma \gamma_{1} \delta_{1} \alpha_{2} \beta_{3}$; eademque via, quam ad stabiliendam formulam (37.) secuti sumus, invenitur $\Sigma \alpha_{1} \delta_{1} \beta_{2} \gamma_{3}=\Sigma \alpha_{1} \delta_{1} \Sigma \beta_{1} \Sigma \gamma_{1}-\Sigma \alpha_{1} \delta_{1} \Sigma \beta_{1} \gamma_{1}-\Sigma \beta_{1} \Sigma \alpha_{1} \gamma_{1} \delta_{1}-\Sigma \gamma_{1} \Sigma \alpha_{1} \beta_{1} \delta_{1}$ $+2 \Sigma \alpha_{1} \beta_{1} \gamma_{1} \delta_{1}$

$\Sigma \beta_{1} \delta_{1} \alpha_{2} \gamma_{3}=\Sigma \beta_{1} \delta_{1} \Sigma \alpha_{1} \Sigma \gamma_{1}-\Sigma \beta_{1} \delta_{1} \Sigma \alpha_{1} \gamma_{1}-\Sigma \alpha_{1} \Sigma \beta_{1} \gamma_{1} \delta_{1}-\Sigma \gamma_{1} \Sigma \alpha_{1} \beta_{1} \delta_{1}$ $+2 \Sigma \alpha_{1} \beta_{1} \gamma_{1} \delta_{1}$

$\Sigma \gamma_{1} \delta_{1} \alpha_{2} \beta_{3}=\Sigma \gamma_{1} \delta_{1} \Sigma \alpha_{1} \Sigma \beta_{1}-\Sigma \gamma_{1} \delta_{1} \Sigma \alpha_{1} \beta_{1}-\Sigma \alpha_{1} \Sigma \beta_{1} \gamma_{1} \delta_{1}-\Sigma \beta_{1} \Sigma \alpha_{1} \gamma_{1} \delta_{1}$ $+2 \Sigma \alpha_{1} \beta_{1} \gamma_{1} \delta_{1}$.

Quibus valoribus in $\left(37^{*}.\right)$ atque $\left(37^{* *}\right.$.) substitutis, derivatur

$$
\begin{gathered}
\Sigma \alpha_{1} \beta_{2} \gamma_{3} \delta_{4}=\Sigma \alpha_{1} \Sigma \beta_{1} \Sigma \gamma_{1} \Sigma \delta_{1}-\Sigma \alpha_{1} \Sigma \beta_{1} \Sigma \gamma_{1} \delta_{1}-\Sigma \alpha_{1} \Sigma \gamma_{1} \Sigma \beta_{1} \delta_{1} \\
-\Sigma \alpha_{1} \Sigma \delta_{1} \Sigma \beta_{1} \gamma_{1}-\Sigma \gamma_{1} \Sigma \delta_{1} \Sigma \alpha_{1} \beta_{1}-\Sigma \beta_{1} \Sigma \delta_{1} \Sigma \alpha_{1} \gamma_{1}-\Sigma \beta_{1} \Sigma \gamma_{1} \Sigma \alpha_{1} \delta_{1} \\
+2 \Sigma \alpha_{1} \Sigma \beta_{1} \beta_{1} \delta_{1}+2 \Sigma \beta_{1} \Sigma \alpha_{1} \gamma_{1} \delta_{1}+2 \Sigma \gamma_{1} \Sigma \alpha_{1} \beta_{1} \delta_{1}+2 \Sigma \delta_{1} \Sigma \alpha_{1} \beta_{1} \gamma_{1} \\
-6 \Sigma \alpha_{1} \beta_{1} \gamma_{1} \delta_{1} .
\end{gathered}
$$

Si singulae summae $\Sigma \alpha_{1}, \Sigma \beta_{1}, \Sigma \gamma_{1}, \Sigma \delta_{1}$ quatuor continent terminos, ita ut sit $\Sigma \alpha_{1}=\alpha_{1}+\alpha_{2}+\alpha_{3}+\alpha_{4}$ etc., pars laeva aequationis (38.), iisdem viginti quatuor terminis composila atque determinans systematis

$$
\begin{array}{llll}
\alpha_{1} & \alpha_{2} & \alpha_{3} & \alpha_{4} \\
\beta_{1} & \beta_{2} & \beta_{3} & \beta_{4} \\
\gamma_{1} & \gamma_{2} & \gamma_{3} & \gamma_{4} \\
\delta_{1} & \delta_{2} & \delta_{3} & \delta_{4}
\end{array}
$$

in eo tantum a determinante differt, quod omnes ejus termini sunt positivi. Unde fluit, partem dextram formulae (38.) evanescere si singulae summae $\Sigma \alpha_{1}, \Sigma \beta_{1}, \Sigma \gamma_{1}, \Sigma \delta_{1}$ tres aut duos contineant terminos, aut modo unum.

Journal d. M. Bd. LIII. Heft 2 . 
Eodem modo, ope formulae (38.) ad reductionem summae $\Sigma \alpha_{1} \beta_{2} \gamma_{3} \delta_{4} \varepsilon_{5}$ adscenditur, et sic porro.

Formulas speciales (26.), (37.), (38.) ill. Binet in 'diario a schola Polytechnica Parisiensi edito (Cah. XVI. pag. 285) publici juris fecit.

IV.

Inter $(n+1)$ radices diversas $u_{1}, u_{2}, \ldots u_{n+1}$ aequationis $2 n^{\text {ti }}$ gradus

$$
\frac{\alpha_{1}}{\left(a_{1}+u\right)^{2}}+\frac{\alpha_{2}}{\left(a_{2}+u\right)^{2}}+\cdots+\frac{\alpha_{n}}{\left(a_{n}+u\right)^{2}}=1
$$

manifesto relatio intercedit a constantibus $\alpha_{1}, \alpha_{2}, \ldots \alpha_{n}$ libera. Quae ut inveniatur $n$ quantitates $\alpha$ inter $(n+1)$ aequationes eliminandae sunt, quarum prima

(40.) $\left|\frac{\alpha_{1}}{\left(a_{1}+u_{1}\right)^{2}}+\frac{\alpha_{2}}{\left(a_{2}+u_{1}\right)^{2}}+\cdots+\frac{\alpha_{n}}{\left(a_{n}+u_{1}\right)^{2}}=1\right|$

brevitatis causa loco totius systematis scribatur. Methodo, quam in art. I. et II. explicavimus, eliminatio ipsarum $\alpha_{1}, \alpha_{2}, \ldots \alpha_{n}$ ad eliminationem $(n-1)$ quantitatum $v_{1}, v_{2}, \ldots v_{n-1}$ e systemate $n$ aequationum

(41.)

$$
\left\{\begin{array}{c}
\frac{1}{a_{1}+v_{1}}+\frac{1}{a_{1}+v_{2}}+\cdots+\frac{1}{a_{1}+v_{n-1}} \\
=\frac{1}{a_{1}+u_{1}}+\frac{1}{a_{1}+u_{2}}+\frac{1}{a_{1}+u_{3}}+\cdots+\frac{1}{a_{1}+u_{n+1}} \\
\frac{1}{a_{2}+v_{1}}+\frac{1}{a_{2}+v_{2}}+\cdots+\frac{1}{a_{2}+v_{n-1}} \\
=\frac{1}{a_{2}+u_{1}}+\frac{1}{a_{2}+u_{2}}+\frac{1}{a_{2}+u_{3}}+\cdots+\frac{1}{a_{2}+u_{n+1}} \\
\frac{1}{a_{n}+v_{1}}+\frac{1}{a_{n}+v_{2}}+\cdots+\frac{1}{a_{n}+v_{n-1}} \\
=\frac{1}{a_{n}+u_{1}}+\frac{1}{a_{n}+u_{2}}+\frac{1}{a_{n}+u_{3}}+\cdots+\frac{1}{a_{n}+u_{n+1}}
\end{array}\right.
$$

reduci potest. Quo ex systemate derivantur $(n-1)$ alia, quorum primum $\frac{n(n-1)}{2}$ aequationes continens ita repraesentari potest:

(42.)

$$
\left|\begin{array}{c}
\frac{1}{\left(a_{1}+v_{1}\right)\left(a_{2}+v_{1}\right)}+\frac{1}{\left(a_{1}+v_{2}\right)\left(a_{2}+v_{2}\right)}+\cdots+\frac{1}{\left(a_{1}+v_{n-1}\right)\left(a_{2}+v_{n-1}\right)} \\
=\frac{1}{\left(a_{1}+u_{1}\right)\left(a_{2}+u_{1}\right)}+\frac{1}{\left(a_{1}+u_{2}\right)\left(a_{2}+u_{2}\right)}+\cdots+\frac{1}{\left(a_{1}+u_{n}\right)\left(a_{2}+u_{n}\right)} \\
+\frac{1}{\left(a_{1}+u_{n+1}\right)\left(a_{2}+u_{n+1}\right)}
\end{array}\right|
$$


7. Jouchimsthal, de quibusd. aequat. quarti et sexti gradus.

secundum autem, $\frac{n(n-1)(n-2)}{1.2 .3}$ aequationes continens,

$$
\left|\begin{array}{c}
\frac{1}{\left(a_{1}+v_{1}\right)\left(a_{2}+v_{1}\right)\left(a_{3}+v_{1}\right)}+\cdots+\frac{1}{\left(a_{1}+v_{n-1}\right)\left(a_{2}+v_{n-1}\right)\left(a_{3}+v_{n-1}\right)} \\
=\frac{1}{\left(a_{1}+u_{1}\right)\left(a_{2}+u_{1}\right)\left(a_{3}+u_{1}\right)}+\cdots+\frac{1}{\left(a_{1}+u_{n}\right)\left(a_{2}+u_{n}\right)\left(a_{3}+u_{n}\right)} \\
\quad+\frac{1}{\left(a_{1}+u_{n+1}\right)\left(a_{2}+u_{n+1}\right)\left(a_{3}+u_{n+1}\right)}
\end{array}\right|
$$

etc., usque ad ultimum systema pervenitur quod unam tantummodo aequationem complectitur

$$
\sum_{v_{1}}^{v_{n-1}} \frac{1}{\left(a_{1}+v_{1}\right)\left(a_{2}+v_{1}\right) \ldots\left(u_{n}+v_{1}\right)}=\sum_{u_{1}}^{u_{n+1}} \frac{1}{\left(a_{1}+u_{1}\right)\left(a_{2}+u_{1}\right) \ldots\left(a_{n}+u_{1}\right)},
$$

quarum aequationum deductio e systemate primitivo (41.) satis obvia est. Jam consideremus summam 1.2.3 $\ldots(n+1)$ terminorum e termino primo permutatione quantitatum $u_{1}, u_{2}, u_{3}, \ldots u_{\xi_{1+1}}$ deducendorum

$$
\Sigma \frac{1}{\left(a_{1}+u_{1}\right)\left(a_{2}+u_{2}\right) \ldots\left(a_{n}+u_{n}\right)} .
$$

Quae summa secundum lemma in III. expositum tanquam summarum simplicium

$$
\left\{\begin{array}{c}
\sum_{u_{1}}^{u_{n+1}} \frac{1}{a_{1}+u_{1}}, \sum \frac{1}{a_{2}+u_{1}}, \ldots \sum \frac{1}{a_{n}+u_{1}} \\
u_{n+1} \frac{1}{\left(a_{1}+u_{1}\right)\left(a_{2}+u_{1}\right)}, \sum \frac{1}{\left(a_{1}+u_{1}\right)\left(a_{3}+u_{1}\right)}, \cdots \sum \sum \frac{1}{\left(a_{n-1}+u_{1}\right)\left(a_{n}+u_{1}\right)} \\
\sum_{u_{1}}^{u_{n+1}} \frac{1}{\left(a_{1}+u_{1}\right)\left(u_{2}+u_{1}\right) \ldots\left(a_{n}+u_{1}\right)}
\end{array}\right.
$$

functio rationalis integra $\boldsymbol{J}$ repraesentari potest. Substituendo autem in $\boldsymbol{J}$ summarum (45.) valores supra inventos, scilicet summas analogas

(46.)

$$
\left\{\begin{array}{c}
\sum_{v_{1}}^{v_{n-1}} \frac{1}{a_{1}+v_{1}}, \sum \frac{1}{a_{2}+v_{1}}, \cdots \sum \frac{1}{a_{n}+v_{1}} \\
v_{n-1}^{n} \frac{1}{\left(a_{1}+v_{1}\right)\left(a_{2}+v_{1}\right)}, \sum \frac{1}{\left(a_{1}+v_{1}\right)\left(a_{3}+v_{1}\right)}, \cdots \sum \frac{1}{\left(a_{n-1}+v_{1}\right)\left(a_{n}+v_{1}\right)} \\
\sum_{v_{1}}^{v_{n-1}} \frac{1}{\left(a_{1}+v_{1}\right)\left(a_{2}+v_{1}\right) \ldots\left(a_{n}+v_{1}\right)}
\end{array}\right.
$$


expressio $J$ in nihilum abit, quum expressiones (46.) (n-1) tantummodo terminis constent. Hinc prodit

Theorema $V$. Designando characteristica $(1,2,3, \ldots n)$ summam $1.2 .3 \ldots n$ terminorum, qui permutatione quantilatum $u_{1}, u_{2}, u_{3}, \ldots u_{n}$ e termino $\frac{1}{\left(a_{1}+u_{1}\right)\left(u_{2}+u_{2}\right) \ldots\left(u_{n}+u_{n}\right)}$ derivantur, inter $(n+1)$ radices diversas $u_{1}, u_{2}, u_{3}, \ldots u_{n+1}$ aequationis $2 n^{\text {ti }}$ gradus

$$
\frac{\alpha_{1}}{\left(a_{1}+u\right)^{2}}+\frac{\alpha_{2}}{\left(a_{2}+u\right)^{2}}+\cdots+\frac{\alpha_{n}}{\left(a_{n}+u\right)^{2}}=1
$$

intercedit relatio

(47.) $(2,3,4, \ldots n+1)+(1,3,4, \ldots n+1)+(1,2,4, \ldots n+1)+\cdots+(1,2,3, \ldots n)=0$ quae aequatio una cum reliquis e (47.) permutatione radicum $u_{1}, u_{2}$,

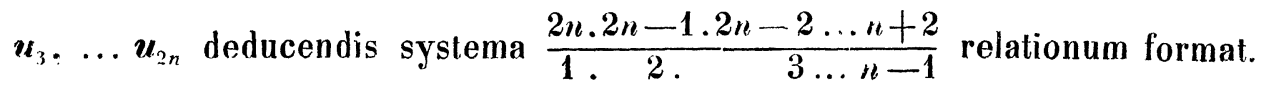

E magna copia relationum quae a formula (39.) derivantur, nunc unam tantummodo eligemus, quae demonstratione muniatur, scilicet sequentem

$$
(1,2,3, \ldots n) \mp(n+1, n+2, n+3, \ldots 2 n)=0,
$$

valente signo superiori vel inferiori prout $n$ par est vel impar (cf. casus speciales (16.) et (33.)). Designemus signo $[1,2,3, \ldots n, n+1], \operatorname{quod} n+1$ indices continet partem laevam formulae (47.), ita ut sit

$[1,2,3, \ldots n, n+1]=(2,3,4, \ldots n+1)+(1,3,4, \ldots n+1)+\cdots+(1,2,3, \ldots n)$.

Distribuantur indices $1,2,3, \ldots 2 n-1,2 n$ in duo systemata, cujus alterum indices $1,2,3, \ldots n$; alterum autem indices $n+1, n+2, n+3, \ldots 2 n$ comprehendat et designetur per $\boldsymbol{A}_{1}$ summa omnium [], quae $n$ indices primi unum autem secundi systematis, per $\boldsymbol{A}_{2}$ summa omnium [], quae $n-1$ indices primi. duos autem secundi systematis contineat, etc., usque ad $\boldsymbol{A}_{n}$ pervenietur, qua littera summam omnium [] designabimus, quae unum indicem primi, $\boldsymbol{n}$ autem indices secundi systematis comprehendunt. Jam dico expressionem

$$
\boldsymbol{A}_{1}-\frac{1}{n-1} \boldsymbol{A}_{2}+\frac{12}{n-1 . n-2} \boldsymbol{A}_{3}-\frac{1.2 .3}{n-1 . n-2 . n-3} \boldsymbol{A}_{4}+\cdots+(-1)^{n-1} \boldsymbol{A}_{n}
$$

substitutis valoribus ipsarum $\boldsymbol{A}_{1}, \boldsymbol{A}_{2}, \ldots \boldsymbol{A}_{n}$ in

$$
n\left\{(1,2,3, \ldots n)+(-1)^{n-1}(n+1, n+2, n+3, \ldots 2 n)\right\}
$$

abire. Manifesto enim expressio $(1,2,3, \ldots n)$ in $n$ terminis solis, quibus $\boldsymbol{A}_{1}$ 
composita est, invenitur; scilicet in

$$
\begin{aligned}
& {[1,2, \ldots n-1, n, n+1], \quad[1,2, \ldots n-1, n, n+2]} \\
& {[1,2, \ldots n-1, n, n+3], \ldots[1,2, \ldots n-1, n, 2 n]}
\end{aligned}
$$

similiter $(n+1, n+2, n+3, \ldots 2 n)$ in $n$ terminis ipsius $A_{n}$ tantum occurrit. Omnes alios terminos in (49.) se mutuo destruere, hoc modo probari potest. Consideremus simplicitatis causa expressionem

$$
(1,2,3, \ldots n-i, n+1, n+2, n+3, \ldots n+i),
$$

quum eadem ratiocinia pro omni expressione () valeant, quae $n-i$ indices primi atque $i$ indices secundi systematis contineat. Quae expressio (51.), ut sine negotio vides, invenitur

$$
\text { in } \begin{gathered}
{[1,2,3, \ldots n-i, n-i+1, n+1, n+2, \ldots n+i]} \\
{[1,2,3, \ldots n-i, n-i+2, n+1, n+2, \ldots n+i]} \\
{[1,2,3, \ldots n-i, n-i+3, n+1, n+2, \ldots n+i]} \\
\vdots \\
\\
\quad[1,2,3, \ldots n-i, n, n+1, n+2, \ldots n+i]
\end{gathered}
$$

atque in $[1,2,3, \ldots n-i, n+1, n+2, n+3, \ldots n+i, n+i+1]$,

$[1,2,3, \ldots n-i, n+1, n+2, n+3, \ldots n+i, n+i+2]$

$[1,2,3, \ldots n-i, n+1, n+2, n+3, \ldots n+i, n+i+3]$,

$[1,2,3, \ldots n-i, n+1, n+2, n+3, \ldots n+i, n+i, 2 n]$.

Jam $i$ expressiones primae seriei ad $\boldsymbol{A}_{i}$ pertinent, itaque in (49.) factore $\pm \frac{1.2 .3 \ldots i-1}{*-1 . n-2 . n-3 \ldots(n-i+1)}$ multiplicatae sunt; atque $(n-i)$ expressiones seriei secundae ad $A_{i+1}$ pertinent, itaque in factorem $\mp \frac{1.2 .3 \ldots i}{n-1 . n-2 \cdot n-3 \ldots n-i}$ ductae sunt. Est igitur in (49.) coefficiens expressionis (51.)

$$
= \pm\left\{i \frac{1.2 \ldots i-1}{n-1 . n-2 \ldots n-i+1}-(n-i) \frac{1.2 \ldots i}{n-1 . n-2 \ldots n-i}\right\}=0, \text { q. e. d. }
$$

Quum autem omnes quantitates [] secundum theorema V. evanescant, summa (49.) sive summa aequivalens (50.) in nihilum abit. Inde prodit formula (48.) sive

Theorema VI. Designando, ut supra, characteristica $(1,2,3, \ldots n)$ summam $1.2 .3 \ldots n$ terminorum $\Sigma_{\frac{1}{\left(a_{1}+u_{1}\right)\left(a_{2}+u_{2}\right) \ldots\left(a_{n}+u_{n}\right)}}^{\text {qui permu- }}$ tatione quantitatum $u_{1}, u_{2}, \ldots u_{n}$ a termino primo derivantur, inter $2 n$ 
radices $u_{1}, u_{2}, \ldots u_{2 n}$ aequationis

$$
\frac{\alpha_{1}}{\left(a_{1}+u\right)^{2}}+\frac{\alpha_{2}}{\left(a_{2}+u\right)^{2}}+\cdots+\frac{\alpha_{n}}{\left(a_{n}+u\right)^{2}}=1
$$

relatio intercedit haecce

$$
\left(48^{*} .\right) \quad(1,2,3, \ldots n)=(-1)^{n}(n+1, n+2, n+3, \ldots 2 n),
$$

ita ut resolutione aequationis $\frac{1}{2} \frac{1.2 .3 \ldots 2 n^{\text {i }}}{(1.2 .3 \ldots n)^{2}}$ gradus $\frac{1.2 .3 \ldots 2 n}{(1.2 .3 \ldots n)^{2}}$ quantitates $(1,2,3, \ldots n)$ ipsae aut earum quadrata inveniri possint.

V.

Jam methodum a nobis adhibitam cum formula e theoria determinantium deducta comparemus. Scribendo

habemus *)

$$
\begin{gathered}
\left(a_{1}+u_{1}\right)\left(a_{1}+u_{2}\right) \ldots\left(a_{1}+u_{n+1}\right)=A_{1} \\
\left(a_{2}+u_{1}\right)\left(a_{2}+u_{2}\right) \ldots\left(a_{2}+u_{n+1}\right)=A_{2} \\
\vdots \\
\left(a_{n}+u_{1}\right)\left(a_{n}+u_{2}\right) \ldots\left(a_{n}+u_{n+1}\right)=A_{n}
\end{gathered}
$$

ubi

$$
\begin{aligned}
& \text { det. }\left\{\frac{1}{\left(a_{1}+u\right)^{2}}, \frac{1}{\left(u_{2}+u\right)^{2}}, \cdots \frac{1}{\left(a_{n}+u\right)^{2}}, \quad 1\right\}=\frac{V}{A_{1}^{2} A_{2}^{2} A_{3}^{2} \ldots A_{n}^{2}} \\
& u=u_{1},=u_{2},=u_{3} \ldots=u_{n+1}
\end{aligned}
$$

(53.) $V=\operatorname{det}\left\{\left\{\left(a_{2}+u\right)^{2}\left(a_{3}+u\right)^{2} \ldots\left(a_{n}+u\right)^{2} ; \ldots\left(a_{1}+u\right)^{2}\left(a_{2}+u\right)^{2} \ldots\left(a_{n-1}+u\right)^{2}\right.\right.$;

$$
\begin{gathered}
\left.\left(a_{1}+u\right)^{2}\left(a_{2}+u\right)^{2} \ldots\left(a_{n}+u\right)^{2}\right\} . \\
u=u_{1},=u_{2},=u_{3} \ldots=u_{n+1}
\end{gathered}
$$

Peracta multiplicatione habemus

$$
\begin{gathered}
\left(\boldsymbol{a}_{2}+\boldsymbol{u}\right)^{2}\left(\boldsymbol{a}_{3}+\boldsymbol{u}\right)^{2} \ldots\left(\boldsymbol{a}_{n}+\boldsymbol{u}\right)^{2}=\lambda_{1}^{(1)}+\lambda_{1}^{(1)} \boldsymbol{u}+\cdots+\lambda_{1}^{(2 n-3)} \boldsymbol{u}^{2 n-3}+\boldsymbol{u}^{2 n-2} \\
\left(\boldsymbol{a}_{1}+\boldsymbol{u}\right)^{2}\left(\boldsymbol{a}_{3}+\boldsymbol{u}\right)^{2} \ldots\left(\boldsymbol{a}_{n}+\boldsymbol{u}\right)^{2}=\lambda_{2}^{(0)}+\lambda_{2}^{(1)} \boldsymbol{u}+\cdots+\lambda_{2}^{(2 n-3)} \boldsymbol{u}^{2 n-3}+\boldsymbol{u}^{2 n-2} \\
\vdots \\
\left(\boldsymbol{a}_{1}+\boldsymbol{u}\right)^{2}\left(\boldsymbol{a}_{2}+\boldsymbol{u}\right)^{2} \ldots\left(\boldsymbol{a}_{n-1}+\boldsymbol{u}\right)^{2}=\lambda_{n}^{(0)}+\lambda_{n}^{(1)} \boldsymbol{u}+\cdots+\lambda_{n}^{(2 n-3)} \boldsymbol{u}^{2 n-3}+\boldsymbol{u}^{2 n-2} \\
\left(\boldsymbol{u}_{1}+\boldsymbol{u}\right)^{2}\left(\boldsymbol{a}_{2}+\boldsymbol{u}\right)^{2} \ldots\left(\boldsymbol{a}_{n-1}+\boldsymbol{u}\right)^{2}\left(\boldsymbol{a}_{n}+\boldsymbol{u}\right)^{2}=\mu^{0}+\mu^{(1)} \boldsymbol{u}+\cdots+\boldsymbol{\mu}^{(2 n-1)} \boldsymbol{u}^{2 n-1}+\boldsymbol{u}^{2 n}
\end{gathered}
$$

ubi quantitates $\lambda$ et $\mu$ ipsarum $a_{1}, a_{2}, \ldots a_{n}$ sunt functionis integrae. Inde sequitur adjumento notissimi theorematis circa determinantium compositionem; ut $V$ tanquam summam $(2 n-1)^{n}(2 n+1)$ determinantium partialium hujusce formae

*) Denotatio determinantiurn, qua utimur, explicatione vix eget. 
7. Joachimsthal, de quibusd: aequat. quarti et sexti gradus.

$$
\text { (54.) v det. }\left|\begin{array}{ccccc}
u_{1}^{\alpha_{1}} & u_{1}^{\alpha_{2}} & \cdots & u_{1}^{\alpha_{n}} & u_{1}^{\alpha_{n+1}} \\
u_{1}^{\alpha_{1}} & u_{2}^{\alpha_{2}} & \cdots & u_{2}^{\alpha_{n}} & u_{2}^{\alpha_{n+1}} \\
& & \vdots & & \\
u_{n+1}^{\alpha_{1}} & u_{n+1}^{\alpha_{2}} & \cdots & u_{n+1}^{\alpha_{n}} & u_{n+1}^{\alpha_{n+1}}
\end{array}\right|
$$

exhiberi possit, ubi $v$ ipsarum $a_{1}, a_{2}, \ldots a_{n}$ functio integra sit. Quum autem determinans (54.) evanescal, quoties duo exponentium $\alpha_{1}, \alpha_{2}, \ldots \alpha_{n}, \alpha_{n+1}$ inter se aequales fiunt, determinans $V$ sive (53.) respectu quantitatum $u_{1}$, $\boldsymbol{u}_{2}, \ldots \boldsymbol{u}_{n+1}$ altiorem ordinem adipisci nequit, quam determinans partialis

$$
\operatorname{det.}\left|\begin{array}{ccccccc}
u_{1}^{n-1} & u_{1}^{n} & u_{1}^{n+1} & \ldots & u_{1}^{2 n-3} & u^{2 n-2} & u_{1}^{2 n} \\
u_{2}^{n-1} & u_{2}^{n} & u_{2}^{n+1} & \ldots & u_{2}^{2 n-3} & u_{2}^{2 n-2} & u_{2}^{2 n} \\
& & & \vdots & & & \\
u_{n+1}^{n-1} & u_{n+1}^{n} & u_{n+1}^{n+1} & \ldots . & u_{n+1}^{2 n-3} & u_{n+1}^{2 n-2} & u_{n+1}^{2 n}
\end{array}\right|
$$

functio $V$ igitur $(n-1)+n+(n+1) \cdots+(2 n-3)+(2 n-2)+2 n=\frac{n(3 n+1)^{\operatorname{tnu}}}{2}$ ordinem non transgreditur. Quum autem $V$ productum ex omnibus ipsarum $u$ differentiis conflatum contineat, scilicet productum

$$
\begin{array}{r}
\Delta\left(u_{1}, u_{2}, u_{3}, \ldots u_{n+1}\right)=\left(u_{1}-u_{2}\right)\left(u_{1}-u_{3}\right) \ldots\left(u_{1}-u_{n}\right)\left(u_{1}-u_{n+1}\right) \\
\left(u_{2}-u_{3}\right) \ldots\left(u_{2}-u_{n}\right)\left(u_{2}-u_{n+1}\right) \\
\vdots \\
\left(u_{n-1}-u_{n}\right)\left(u_{n-1}-u_{n+1}\right) \\
\left(u_{n}-u_{n+1}\right)
\end{array}
$$

quod $\frac{n(n+1)^{\mathrm{ti}}}{2}$ ordinis est, manifesto poni potest

$$
\text { (56.) } V=\Delta\left(u_{1}, u_{2}, u_{3}, \ldots u_{n+1}\right) V_{1},
$$

denotando per $V_{1}$ functionem, quae $n^{2 \text { tum }}$ ordinem non transgrediatur.

Consideremus expressionem supra signo $[1,2,3, \ldots n+1]$ notatam, scilicet summam 1.2.3..n +1 terminorum qui e termino $\frac{1}{\left(a_{1}+u_{1}\right)\left(a_{2}+u_{2}\right) \ldots\left(a_{n}+u_{n}\right)}$ permutatione radicum $u_{1}, u_{2}, u_{3}, \ldots u_{n+1}$ derivantur. Quae summa manifesto in $\frac{U}{A_{1} A_{2} A_{3} \ldots A_{n}}$ transformari potest, ubi $U$ est functio, respectu ipsarum $u_{1}, u_{2}, \ldots u_{n+1}$ non minoris quam $n^{2 \text { ti }}$ ordinis, quae factores $u_{1}-u_{2}$, $u_{1}-u_{3}, \ldots u_{n}-u_{n+1}$ non contineat. Quum autem eliminatione quantitatum $\alpha$ e systemate $(n+1)$ aequationum

$$
\left|\begin{array}{c}
\frac{\alpha_{1}}{\left(a_{1}+u\right)^{2}}+\frac{\alpha_{2}}{\left(a_{2}+u\right)^{2}}+\cdots+\frac{\alpha_{n}}{\left(a_{n}+u\right)^{2}}-1=0 \\
u=u_{1},=u_{2}, \ldots,=u_{n+1}
\end{array}\right|
$$


methocio nota aequatio $V=0$, sive $\mathcal{L}\left(u_{1}, u_{2}, \ldots u_{n+1}\right) V_{1}=0$, nostra autem methodo aequatio $U=0$, obtinetur; expressiones $V_{1}$ atque $U$, quum posterior priorem, quae, ut facile probatur, identice non evanescit, metiri debeat, nisi factore a solis quantitatibus $a_{1}, a_{2}, \ldots a_{n}$ dependenti differre nequeunt. Habemus igitur formulam

$$
\text { det. } \begin{aligned}
\left\{\frac{1}{\left(a_{1}+u\right)^{2}}, \frac{1}{\left(u_{2}+u\right)^{2}}, \cdots\right. & \left.\frac{1}{\left(a_{n}+u\right)^{2}}, 1\right\} \\
u=u_{1},=u_{2}, \ldots, & =u_{n+1} \\
& =\varrho[1,2,3 \ldots n+1] \frac{d\left(u_{1}, u_{2}, \ldots u_{n+1}\right)}{A_{1} \cdot A_{2} \cdot A_{3} \ldots A_{n}}
\end{aligned}
$$

denotando per $\varrho$ functionem rationalem ipsarum $a_{1}, a_{2}, \ldots a_{n}$.

\section{VI.}

Quum quinque abhinc annis theoremata eaque demonstrandi methodos, quae in articulis praecedentibus leguntur, invenissem, a determinatione generali ipsius o propter calculorum, in quos incideram, prolixitatem abhorrui; pro casibus autem specialibus $n=2,=3$, qui soli in illis applicationibus geometricis occurrunt, de quibus postea disseremus, valores ipsius $\varrho$ sine magno labore erui potuerunt. Jam ut de novo quaestionem intacte relictam susciperem, theorema pulcherrimum me commovit, a $\mathrm{cl}^{\circ}$. Borchardt inventum, atque, cum luculenta demonstratione, in commentatione de functionibus symmetricis illustri Academiae Berolinensi praeterito anno tradita publicalum *). Propositio, inter elegantissimas, quae de determinantibus traduntur, formulas censenda hoc modo exhiberi potest

$$
\text { 8.) } \begin{aligned}
& \operatorname{det}\left\{\frac{1}{\left(u_{1}+u\right)^{2}}, \frac{1}{\left(a_{2}+u\right)^{2}}, \cdots \frac{1}{\left(a_{n}+u\right)^{2}}\right\} \\
& \text { det. }\left\{\frac{1}{a_{1}+u}, \frac{1}{a_{2}+u}, \cdots \frac{1}{a_{n}+u}\right\} \\
&=\Sigma \frac{u_{1},=u_{2}, \ldots,=u_{n}}{\left(a_{1}+u_{1}\right)\left(a_{2}+u_{2}\right) \ldots\left(a_{n}+u_{n}\right)}=(1,2,3, \ldots n)
\end{aligned}
$$

(De vi characteristicae $(1,2,3, \ldots n)$ vide theorema V.).

Permagna hujus formulae atque relationis (57.) similitudo ad novas disquisitiones instituendas me induxit, et adjumento nonnullorum artificiorum, quae olim neglexeram, ad formulam illi (58.) omnino analogam perveni. Quas disquisitiones hic addere non inutile fore credo, ne articuli praecedentis evo-

*) Cf. Relat. menstr. Acad. Berol. pro anno 1855. 
lutiones incompletae maneant; solutionis autem merita, si quae adsint, relationis (58.) sagacissimo auctori sunt attribuenda.

Multiplicando aequationem (57.) per productum $\left(a_{1}+u_{1}\right)^{2}\left(a_{2}+u_{2}\right)^{2} \ldots$ $\left(a_{n}+u_{n}\right)^{2}$ atque ponendo deinde $u_{1}=-a_{1}, u_{2}=-a_{2}, \ldots u_{n}=-a_{n}$ valor ipsius $\varrho$ facile eruitur. Quibus calculis enim pars laeva aequationis (57.) sive

mutatur in

$$
\operatorname{det.}\left|\begin{array}{ccccc}
\frac{1}{\left(a_{1}+u_{1}\right)^{2}} & \frac{1}{\left(a_{2}+u_{1}\right)^{2}} & \cdots & \frac{1}{\left(a_{n}+u_{1}\right)^{2}} & 1 \\
\frac{1}{\left(a_{1}+u_{2}\right)^{2}} & \frac{1}{\left(a_{2}+u_{2}\right)^{2}} & \cdots & \frac{1}{\left(a_{n}+u_{2}\right)^{2}} & 1 \\
\frac{1}{\left(a_{1}+u_{n}\right)^{2}} & \frac{1}{\left(a_{2}+u_{n}\right)^{2}} & \cdots & \frac{1}{\left(a_{n}+u_{n}\right)^{2}} & 1 \\
\frac{1}{\left(a_{1}+\frac{1}{\left.u_{n+1}\right)^{2}}\right.} & \frac{1}{\left(a_{2}+\frac{1}{\left.u_{n+1}\right)^{2}}\right.} & \cdots & \frac{1}{\left(a_{n}+u_{n+1}\right)^{2}} & 1
\end{array}\right|
$$

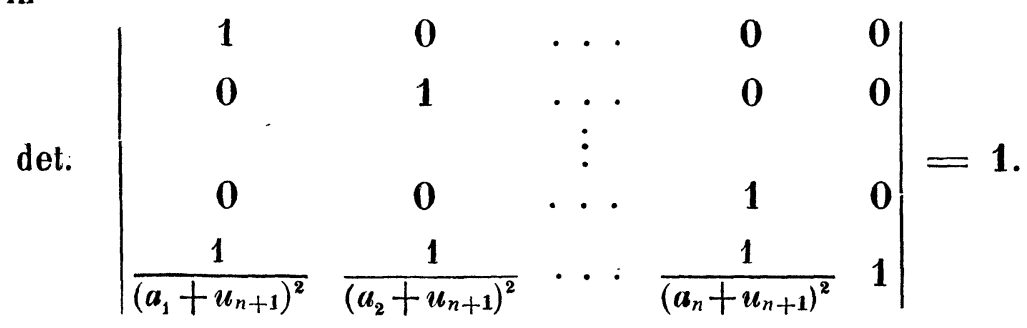

In secundo membro aequationis (57.)

$\left.\Delta\left(u_{1}, u_{2}, \ldots u_{n+1}\right)=\left(u_{1}-u_{n+1}\right)\left(u_{2}-u_{n+1}\right) \ldots\left(u_{n}-u_{n+1}\right) \Delta\left(u_{1}, u_{2}, u_{3}, \ldots u_{n}\right)^{*}\right)$ transit in $(-1)^{n} .\left(a_{1}+u_{n+1}\right)\left(a_{2}+u_{n+1}\right) \ldots\left(a_{n}+u_{n+1}\right)(-1)^{\frac{n(n-1)}{2}} \Delta\left(a_{1}, a_{2}, \ldots a_{n}\right)$; atque $\frac{[1,2,3, \ldots n+1]}{A_{1} A_{2} A_{3} \ldots A_{n}}$, multiplicatione facta, substitutisque valoribus, quos ipsis $u_{1}, u_{2}, \ldots u_{n}$ tribuimus, mutatur in fractionem, cujus numerator unitas, denominator autem

$$
\begin{aligned}
= & \left(a_{1}-a_{2}\right)\left(a_{1}-a_{3}\right) \ldots\left(a_{1}-a_{n}\right)\left(a_{1}+u_{n+1}\right) \\
& \times\left(a_{2}-a_{1}\right)\left(a_{2}-a_{3}\right) \ldots\left(a_{2}-a_{n}\right)\left(a_{2}+u_{n+1}\right) \\
& \times\left(a_{3}-a_{1}\right)\left(a_{3}-a_{2}\right) \ldots\left(a_{3}-a_{n}\right)\left(a_{3}+u_{n+1}\right) \\
& \times\left(a_{n}-a_{1}\right)\left(a_{n}-a_{2}\right) \ldots\left(a_{n}-a_{n-1}\right)\left(a_{n}+u_{n+1}\right) \\
=(-1)^{\frac{n(n-1)}{2}} & \frac{1}{\Delta\left(a_{1}, a_{2}, \ldots a_{n}\right)^{2}\left(a_{1}+u_{n+1}\right)\left(a_{2}+u_{n+1}\right) \cdots\left(a_{n}+u_{n+1}\right)} .
\end{aligned}
$$

*) In producto differentiarum, quod signo $\Delta$ denotamus, singulae differentiae $u_{\alpha}-u_{\beta}$ ita sumendae sunt ut $\alpha<\beta$.

Journal f. d. M. Bd. LIII. Heft 2. 
Quibus valoribus substitutis aequatio (57.) transit in

$$
1=\varrho \frac{(-1)^{n}}{\Delta\left(a_{1}, a_{2}, \ldots a_{n}\right)}, \quad \text { unde } \varrho=(-1)^{n} \Delta\left(a_{1}, a_{2}, \ldots a_{n}\right)
$$

Hinc prodit denique e (57.)

$$
\begin{gathered}
\text { (59.) det. }\left\{\frac{1}{\left(a_{1}+u\right)^{2}}, \frac{1}{\left(a_{2}+u\right)^{2}}, \cdots \frac{1}{\left(a_{n}+u\right)^{2}}, 1\right\} \\
u=u_{1},=u_{2}, \ldots=u_{n+1} \\
=(-1)^{n} \frac{\Delta\left(a_{1}, a_{2}, \ldots a_{n}\right) \Delta\left(u_{1}, u_{2}, \ldots u_{n+1}\right)}{A_{1} A_{2} A_{3} \ldots A_{n}}[1,2,3, \ldots n+1] .
\end{gathered}
$$

Simili modo ut supra, videlicet comparatione dimensionum, invenitur esse

(60.) det. $\left\{\frac{1}{a_{1}+u}, \frac{1}{a_{2}+u}, \cdots \frac{1}{a_{n}+u}, 1\right\}=\frac{\sigma \Delta\left(u_{1}, u_{2}, \ldots u_{n+1}\right)}{A_{1} A_{2} A_{3} \ldots A_{n}}$

$$
u=u_{1},=u_{2}, \ldots=u_{n+1}
$$

ubi $\sigma$ ipsarum $a_{1}, a_{2}, \ldots a_{n}$ sit functio rationalis. Multiplicando aequationem praecedentem per $\left(a_{1}+u_{1}\right)\left(a_{2}+u_{2}\right) \ldots\left(a_{n}+u_{n}\right)$, atque ponendo $u_{1}=-a_{1}$, $\boldsymbol{u}_{2}=-a_{2}, \ldots u_{n}=-a_{n}$, obtinemus $\sigma=\varrho=(-1)^{n} \Delta\left(a_{1}, a_{2}, \ldots a_{n}\right)$, unde

$$
\begin{gathered}
\text { (61.) det. }\left\{\frac{1}{a_{1}+u}, \frac{1}{a_{2}+u}, \cdots \frac{1}{a_{n}+u}, 1\right\} \\
u=u_{1},=u_{2},=u_{3}, \ldots=u_{n+1} \\
=(-1)^{n} \frac{\Delta\left(a_{1}, a_{2}, a_{3}, \ldots a_{n}\right) \Delta\left(u_{1}, u_{2}, u_{3}, \ldots u_{n+1}\right)}{A_{1} A_{2} A_{3} \ldots A_{n}} .
\end{gathered}
$$

E combinatione aequationum (59.) et (61.) prodit

$$
\begin{gathered}
\text { det. }\left\{\frac{1}{\left(a_{1}+u\right)^{2}}, \frac{1}{\left(a_{2}+u\right)^{2}}, \cdots \frac{1}{\left(a_{n}+u\right)^{2}}, 1\right\} \\
\text { det. }\left\{\frac{1}{a_{1}+u}, \frac{1}{a_{2}+u}, \cdots \frac{1}{a_{n}+u}, 1\right\} \\
u=u_{1},=u_{2},=u_{3}, \ldots=u_{n+1}
\end{gathered}=[1,2,3, \ldots n+1] .
$$

Faciendo $u_{n+1}=$ quantitati infinite magnae, aequatio (62.) in relationem a cl. Borchardt inventam transit, scilicet in

$$
\begin{aligned}
& \text { (58.) } \frac{\operatorname{det}\left\{\frac{1}{\left(a_{1}+u\right)^{2}}, \frac{1}{\left(a_{2}+u\right)^{2}}, \cdots \frac{1}{\left(a_{n}+u\right)^{2}}\right\}}{\operatorname{det}\left\{\frac{1}{a_{1}+u}, \frac{1}{a_{2}+u}, \cdots \frac{1}{a_{n}+u}\right\}} \\
& u=u_{1},=u_{2}, \ldots=u_{n} \\
& =(1,2,3, \ldots n)=\Sigma \frac{1}{\left(a_{1}+u_{1}\right)\left(a_{2}+u_{2}\right) \ldots\left(a_{n}+u_{n}\right)} .
\end{aligned}
$$

Simili modo ut e formula (62.) relationem (58.) deduximus, a posteriori ad 
7. Joachimsthal, de quibusd. aequat. quarti et sexti gradus.

priorem calculo persimplici perveniri potest. Insuper relationis (58.) demonstratio exstat directa, quae prorsus similibus, atque supra adhibita sunt, nititur ratiociniis.

VII.

Ad applicationes geometricas transeamus, quae casibus $n=2$ et $n=3$ respondent. Puncta curvae

$$
\text { (1.) } \frac{x^{2}}{a}+\frac{y^{2}}{b}-1=0,
$$

quorum normales per datum punctum $(\xi, \eta)$ transeunt, ut supra vidimus, formulis data sunt

$$
\left(2^{*} .\right) \quad x_{1}=\frac{a \xi}{a+u_{1}}, \quad y=\frac{b \eta}{b+u_{1}},
$$

littera $u_{1}$ denotante radicem aequationis

$$
\text { (3.) } \frac{a \xi^{2}}{(a+u)^{2}}+\frac{b \eta^{2}}{(b+u)^{2}}=1 \text {. }
$$

Statuendo porro $x_{2}=\frac{a \xi}{a+u_{2}}, y_{2}=\frac{b \eta}{b+u_{2}}$, etc., aequatio (14.) sive $[1,2,3]=0$, transformari potest in

(63.) $\quad x_{2} y_{3}+x_{3} y_{2}+x_{1} y_{3}+x_{3} y_{1}+x_{1} y_{2}+x_{2} y_{1}=0$.

Si igitur puncta $\left(x_{1} y_{1}\right),\left(x_{2} y_{2}\right),\left(x_{3} y_{3}\right)$ conicae (1.) aequationi (63.) satisfaciunt, normales curvae in his tribus punctis erectae per idem punctum transeunt, sive

puncta conicae (1.), quorum normales cum normalibus punctorum conicae $\left(x_{1}, y_{1}\right),\left(x_{2}, y_{2}\right)$ in idem punctum concurrunt, in recta

(64.) $\quad x\left(y_{1}+y_{2}\right)+y\left(x_{1}+x_{2}\right)+x_{1} y_{2}+x_{2} y_{1}=0$

sita sunt.

Considerationibus geometricis ductus constructionem rectae (64.) hancce dedimus (cf. Comment. de normalibus ellipsis atque ellipsoidae, t. XXVI hujus diarii). Sit $(p, q)$ polus rectae puncta $\left(x_{1}, y_{1}\right),\left(x_{2}, y_{2}\right)$ jungentis; habetur

$$
\begin{array}{ll}
\frac{p x_{1}}{a}+\frac{q y_{1}}{b}=1, & \frac{x_{1}^{2}}{a}+\frac{y_{1}^{2}}{b}=1, \\
\frac{p x_{2}}{a}+\frac{q y_{2}}{b}=1, & \frac{x_{2}^{2}}{a}+\frac{y_{2}^{2}}{b}=1,
\end{array}
$$

ergo

$$
\frac{p}{a}=\frac{y_{2}-y_{1}}{x_{1} y_{2}-x_{2} y_{1}}, \quad \frac{1}{a}=\frac{y_{2}^{2}-y_{1}^{2}}{x_{1}^{2} y_{2}^{2}-x_{2}^{2} y_{1}^{2}}
$$


unde (65.)

sive (66.)

$$
p=\frac{x_{1}^{2} y_{2}^{2}-x_{2}^{2} y_{1}^{2}}{x_{1} y_{2}-x_{2} y_{1}} \frac{y_{2}-y_{1}}{y_{2}^{2}-y_{1}^{2}}
$$

$$
p=\frac{x_{1} y_{2}+x_{2} y_{1}}{y_{1}+y_{2}} \text { atque similiter } q=\frac{x_{1} y_{2}+x_{2} y_{1}}{x_{1}+x_{2}}
$$

Hinc prodit theorema sequens, quod in commentatione supra laudata invenitur:

Designando per $\boldsymbol{p}$ et $\boldsymbol{q}$ coordinatas rectangulares poli lineae rectae, quae puncta $h_{1}, h_{2}$ sectionis conicae $\frac{x^{2}}{a}+\frac{y^{2}}{b}-1=0$ jungit, recta $\frac{x}{p}+\frac{y}{q}+1=0$ conicam in duabus aliis punctis $h_{3}, h_{4}$ (sive realibus, sive imaginariis), secabit ita ut normales curvae in punctis $h_{1}, h_{2}, h_{3}, h_{4}$ ductae in eodem puncto concurrant.

Jam ad superficiem $\frac{x^{2}}{a}+\frac{y^{2}}{b}+\frac{z^{2}}{c}-1=0$ progrediamur.

Supponamus a puncto quodam $h$ ad superficiem sex normales ductas esse $h h_{1}, h h_{2}, h h_{3}, h h_{4}, h h_{5}, h h_{6}$. Designando per $\xi, \eta, \zeta$ coordinatas ipsius $h$, per $x_{1}, y_{1}, z_{1}$ coordinatas puncti $h_{1}$, quod in superficie ipsa situm est, etc. habetur

$$
\begin{aligned}
& x_{1}=\frac{a \xi}{a+u_{1}}, \quad y_{1}=\frac{b \eta}{b+u_{1}}, \quad z_{1}=\frac{c \zeta}{c+u_{1}}, \\
& x_{2}=\frac{a \xi}{a+u_{2}}, \quad y_{2}=\frac{b \eta}{b+u_{2}}, \quad z_{2}=\frac{c \zeta}{c+u_{2}} \text {, etc. }
\end{aligned}
$$

ubi $u_{1}, u_{2}, \ldots u_{6}$ sunt radices aequationis

$$
\frac{a \xi^{2}}{(a+u)^{2}}+\frac{b \eta^{2}}{(b+u)^{2}}+\frac{\dot{c} \zeta^{2}}{(c+u)^{2}}-1=0
$$

Quibus valoribus relatio (31.) transit in

$$
\begin{aligned}
& x_{2} y_{3} z_{4}+x_{2} y_{4} z_{3}+x_{3} y_{4} z_{2}+x_{3} y_{2} z_{4}+x_{4} y_{2} z_{3}+x_{4} y_{3} z_{2} \\
+ & x_{1} y_{3} z_{4}+x_{1} y_{4} z_{3}+x_{3} y_{4} z_{1}+x_{3} y_{1} z_{4}+x_{4} y_{1} z_{3}+x_{4} y_{3} z_{1} \\
+ & x_{1} y_{2} z_{4}+x_{1} y_{4} z_{2}+x_{2} y_{4} z_{1}+x_{2} y_{1} z_{4}+x_{4} y_{1} z_{2}+x_{4} y_{2} z_{1} \\
+ & x_{1} y_{2} z_{3}+x_{1} y_{3} z_{2}+x_{2} y_{3} z_{1}+x_{2} y_{1} z_{3}+x_{3} y_{1} z_{2}+x_{3} y_{2} z_{1}=0
\end{aligned}
$$

quae est conditio necesssaria (neutiquam autem sufficiens), si normales in punctis snperficiei $\left(x_{1}, y_{1}, z_{1}\right),\left(x_{2}, y_{2}, z_{2}\right),\left(x_{3}, y_{3}, z_{3}\right),\left(x_{4}, y_{4}, z_{4}\right)$ ductae per idem punctum $h$ transeunt. Formula (67.) etiam hoc modo enuntiari potest:

Si normales in punctis $\left(x_{1}, y_{1}, z_{1}\right),\left(x_{2}, y_{2}, z_{2}\right),\left(x_{3}, y_{3}, z_{3}\right)$ superficiei $\frac{x^{2}}{a}+\frac{y^{2}}{b}+\frac{z^{2}}{c}-1=0$ in eodem puncto $h$ concurrunt, tria alia puncta, 
7. Joachimsthal, de quibusd. aequat. quarti et sexti gradus.

quorum normales per $h$ transeunt, in plano

(68.)

$$
\begin{aligned}
& x\left\{y_{2} z_{3}+y_{3} z_{2}+y_{1} z_{3}+y_{3} z_{1}+y_{1} z_{2}+y_{2} z_{1}\right\} \\
+ & y\left\{z_{2} x_{3}+z_{3} x_{2}+z_{1} x_{3}+z_{3} x_{1}+z_{1} x_{2}+z_{2} x_{1}\right\} \\
+ & z\left\{x_{2} y_{3}+x_{3} y_{2}+x_{1} y_{3}+x_{3} y_{1}+x_{1} y_{2}+x_{2} y_{1}\right\} \\
+ & x_{1} y_{2} z_{3}+x_{1} y_{3} z_{2}+x_{2} y_{1} z_{3}+x_{2} y_{3} z_{1}+x_{3} y_{1} z_{2}+x_{3} y_{2} z_{1}=0
\end{aligned}
$$

sita sunt.

Sit $(\mu, q, r)$ polus plani, tria puncta $\left(x_{1}, y_{1}, z_{1}\right),\left(x_{2}, y_{2}, z_{2}\right),\left(x_{3}, y_{3}, z_{3}\right)$ jungentis, habentur sex aequationes

$$
\begin{array}{ll}
\frac{p x_{1}}{a}+\frac{q y_{1}}{b}+\frac{r z_{1}}{c}=1 ; & \frac{x_{1}^{2}}{a}+\frac{y_{1}^{2}}{b}+\frac{z_{1}^{2}}{c}=1 . \\
\frac{p x_{2}}{a}+\frac{q y_{2}}{b}+\frac{r z_{2}}{c}=1 ; & \frac{x_{2}^{2}}{a}+\frac{y_{2}^{2}}{b}+\frac{z_{2}^{2}}{c}=1 . \\
\frac{p x_{3}}{a}+\frac{q y_{3}}{b}+\frac{r z_{3}}{c}=1 ; & \frac{x_{3}^{2}}{a}+\frac{y_{3}^{2}}{b}+\frac{z_{3}^{2}}{c}=1 ;
\end{array}
$$

unde

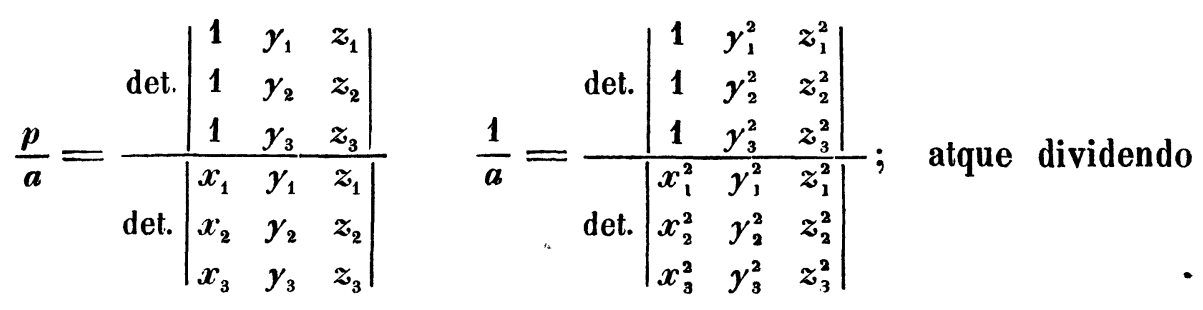

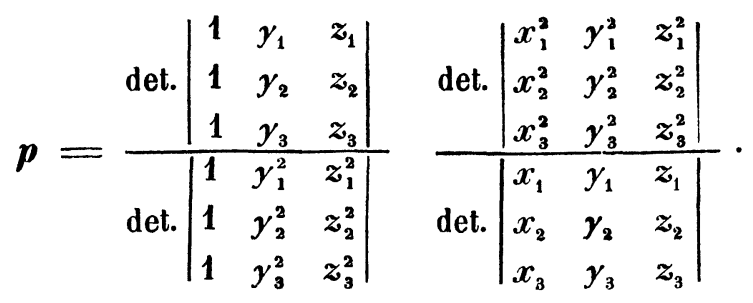

Generaliter loquendo hic valor ipsius $p$ reductionem non admittit. Substitutis autem valoribus

$$
x_{1}=\frac{a \xi}{a+u_{1}}, \quad y_{1}=\frac{b \eta}{b+u_{1}}, \quad z_{1}=\frac{c \zeta}{c+u_{1}} \text { etc. }
$$

habetur secundum formulam (62.) 


$$
\begin{aligned}
& \frac{\operatorname{det} .\left|\begin{array}{lll}
1 & y_{1}^{2} & z_{1}^{2} \\
1 & y_{2}^{2} & z_{2}^{2} \\
1 & y_{3}^{2} & z_{3}^{2}
\end{array}\right|}{\operatorname{det}\left|\begin{array}{lll}
1 & y_{1} & z_{1} \\
1 & y_{2} & z_{2} \\
1 & y_{3} & z_{3}
\end{array}\right|}=b c \eta \zeta \frac{\operatorname{det} .\left\{1, \frac{1}{(b+u)^{2}}, \frac{1}{(c+u)^{2}}\right\}}{\operatorname{det}\left\{\begin{array}{c}
\left.1, \frac{1}{(b+u)}, \frac{1}{(c+u)}\right\} \\
u=u_{1},=u_{3},=u_{3}
\end{array}\right.} \\
& =b c \eta \zeta\left\{\frac{1}{\left(b+u_{2}\right)\left(c+u_{3}\right)}+\frac{1}{\left(b+u_{3}\right)\left(c+u_{2}\right)}+\cdots+\frac{1}{\left(b+u_{2}\right)\left(c+u_{1}\right)}\right\} \\
& =y_{2} z_{3}+y_{3} z_{2}+y_{3} z_{1}+y_{1} z_{3}+y_{1} z_{2}+y_{2} z_{1}
\end{aligned}
$$

atque secundum (58.),

$$
\begin{gathered}
\operatorname{det} .\left|\begin{array}{lll}
x_{1}^{2} & y_{1}^{2} & z_{1}^{2} \\
x_{2}^{2} & y_{2}^{2} & z_{2}^{2} \\
x_{3}^{2} & y_{3}^{2} & z_{3}^{2}
\end{array}\right| \\
\operatorname{det.}\left|\begin{array}{lll}
x_{1} & y_{1} & z_{1} \\
x_{2} & y_{2} & z_{2} \\
x_{3} & y_{3} & z_{3}
\end{array}\right|
\end{gathered}
$$

itaque

$$
p=\frac{x_{1} y_{2} z_{3}+x_{1} y_{3} z_{2}+x_{2} y_{1} z_{3}+x_{2} y_{3} z_{1}+x_{3} y_{1} z_{2}+x_{3} y_{2} z_{1}}{y_{2} z_{3}+y_{3} z_{2}+y_{1} z_{3}+y_{3} z_{1}+y_{1} z_{2}+y_{2} z_{1}}
$$

perinde ipsarum $q$ et $\boldsymbol{r}$ valores eruendi sunt. Ratione igitur habita aequationis (68.) hanc propositionem nacti sumus:

Sint $h_{1}, h_{2}, h_{3}$ tria puncta superficiei $\frac{x^{2}}{a}+\frac{y^{2}}{b}+\frac{z^{2}}{c}-1=0$, quorum normales in eodem puncto $h$ concurrunt; designando per $p, q$ et $r$ coordinatas poli plani $h_{1} h_{2} h_{3}$, tria alia puncta $h_{4}, h_{5}, h_{6}$ superficiei, quorum normales per $h$ transeunt, in plano

$$
\frac{x}{p}+\frac{y}{q}+\frac{z}{r}+1=0
$$

sita sunt.

Permultas alias propositiones geometricas e formulis praecedentibus deducendas, quippe quae a nostro fine alienae sunt, nunc praetereamus.

Carlottae-fontibus, m. Sept. 1856. 